\title{
On Higher Order Approximations for Hermite-Gaussian Functions and Discrete Fractional Fourier Transforms
}

\author{
Çagatay Candan
}

\begin{abstract}
Discrete equivalents of Hermite-Gaussian functions play a critical role in the definition of a discrete fractional Fourier transform. The discrete equivalents are typically calculated through the eigendecomposition of a commutator matrix. In this letter, we first characterize the space of DFT-commuting matrices and then construct matrices approximating the Hermite-Gaussian generating differential equation and use the matrices to accurately generate the discrete equivalents of Hermite-Gaussians.
\end{abstract}

Index Terms-Commuting matrices, fractional Fourier transforms, Hermite-Gaussian functions.

\section{INTRODUCTION}

A CCURATE and efficient discretization of operators defined in a continuum is a necessity with the advent of digital computational tools. A successful discretization should inherit as many operational properties of the continuous operator as possible and at the same time should have an efficient means of calculation. The prime example for a successful discretization is the discrete Fourier transform (DFT). Following the Fourier transform, the cosine transform and other related transforms such as sine, Hartley, etc. have been discretized in the 1970s. In the late 1990s, attention focused on the discrete version of the fractional Fourier transform [1].

The discretization of a fractional Fourier transform is particularly challenging due to the difficulties with its highly oscillatory kernel. To date, various methods have been proposed. Among these methods, discretization by the commutator matrix has been found useful since this method preserves many properties of continuous transform and approximates the transform quite closely.

In this letter, we present a set of commuting matrices that provide finer approximations to Hermite-Gaussian functions. The commuting matrices here have been first discussed in [2] by mentioning that some higher order matrices can be utilized when it is desired to simulate the continuous transforms very accurately. In this letter, we show that higher order commuting matrices indeed provide much better approximations than the original matrix [2] and also outperform some other proposed matrices recently reported in the literature [3], [4].

Manuscript received January 10, 2007; revised March 13, 2007. The associate editor coordinating the review of this manuscript and approving it for publication was Dr. Shahram Shahbazpanahi.

The author is with the Department of Electrical Engineering, Middle East Technical University, Ankara, Turkey (e-mail: ccandan@metu.edu.tr).

Digital Object Identifier 10.1109/LSP.2007.898354
This letter is organized as follows: Section II introduces Hermite-Gaussian functions and briefly establishes the connection between these functions and the fractional Fourier transform. Section III characterizes the space of matrices commuting with the DFT matrix. The results of this section help us to conduct a search on DFT-commuting matrices whose eigenvectors are good approximations to the samples of Hermite-Gaussians. Section IV is on the derivation of commuting matrices generating discrete equivalents of Hermite-Gaussians. Section IV compares the method with the existing schemes in the literature.

\section{Hermite-Gaussian Functions AND FOURIER TRANSFORM}

Discretization of a differential equation requires a careful practice since the choice of sampling locations (meshpoint or midpoint), boundary conditions, and approximation order may lead to very different finite dimensional equivalents. For example, the solution of the differential equation $-f^{\prime \prime}(x)=\lambda f(x)$, with the boundary conditions $f^{\prime}(0)=0$ and $f^{\prime}(\pi)=0$, results in $\cos (k x)$ functions, which form a set of orthonormal functions in $[0, \pi],\left(\lambda=k^{2}\right)$. By replacing the second derivative with second differencing and substituting the suitable boundary conditions for chosen discretization, we can form a difference equation whose eigenvectors would be the discrete equivalents of $\cos (k x)$ functions. In [5], it has been illustrated that the multiplicity of ways in expressing boundary conditions leads to four different discrete equivalents for $\cos (k x)$ (each of which is formed by differently sampling $\cos (k x)$ in $[0, \pi])$. The most important equivalent among the four is the DCT-2, which is used in JPEG image compression. The multiplicity of discrete equivalents for a continuous problem is an inherent difficulty of the discretization process and should be carefully examined.

The differential equation generating Hermite-Gaussian functions is given as follows:

$$
\frac{d^{2} f(t)}{d t^{2}}-4 \pi t^{2} f(t)=2 \pi(2 n+1) f(t) .
$$

We prefer to express the left-hand side of (1) in the operator notation as

$$
\mathcal{S}\{f(t)\}=\lambda f(t)
$$

where $f(t)$ corresponds to the eigenfunction of the operator $\mathcal{S}$. In [2], it has been shown that $\mathcal{S}=\mathcal{D}^{2}+\mathcal{F D}^{2} \mathcal{F}^{-1}$, where $\mathcal{D}$ 
is the derivative operator and $\mathcal{F}$ is the Fourier transformation operator defined as $\mathcal{F}\{f(t)\}=\int_{-\infty}^{\infty} f\left(t^{\prime}\right) e^{-j 2 \pi t t^{\prime}} d t^{\prime}$. The importance of Hermite-Gaussians stems from their eigen-relation with the Fourier transform. The Hermite-Gaussian functions form a complete, orthogonal eigenfunction set of the Fourier transform. This result has been shown by the commutation property of $\mathcal{S}$ and $\mathcal{F}$ in [2]

In this letter, we would like to find matrices approximating operator $\mathcal{S}$ and commuting with the DFT matrix. To this end, the $\mathbf{S}$ matrix first proposed in [6] has been utilized in [2]. Recently, the $\mathbf{T}$ matrix first proposed in [7] has been suggested in [3]. Here, we present additional matrices with higher approximation powers and show that these matrices provide much more accurate representations. To illustrate the performance of these matrices, we study the general structure of matrices commuting with the DFT matrix first.

\section{SPACE OF COMMUTING MATRICES}

Matrices commuting with the DFT matrix $(\mathbf{F})$ form a linear space [8, p. 416]. Some elements of this space are the identity matrix, the all zero matrix, the $\mathbf{F}$ matrix (and its powers), the $\mathbf{S}$ matrix of [6], and the $\mathbf{T}$ matrix of [7].

In the present section, we show that any matrix $\mathbf{K}$ commuting with $\mathbf{F}$ can be expressed as follows:

$$
\mathbf{K}=\mathbf{M}+\mathbf{F M F}^{-1}+\mathbf{F}^{2} \mathbf{M F} \mathbf{F}^{-2}+\mathbf{F}^{3} \mathbf{M} \mathbf{F}^{-3}
$$

where $\mathbf{M}$ is a matrix of matching dimensions, generating the commutator matrix $\mathbf{K}$.

Theorem 1: The matrix $\mathbf{K}$ and DFT matrix $\mathbf{F}$ commute.

Proof: Multiplying $\mathbf{K}$ from left and right by $\mathbf{F}$ and $\mathbf{F}^{-1}$, we get

$\mathbf{F K F} \mathbf{F}^{-1}=\mathbf{F M} \mathbf{F}^{-1}+\mathbf{F}^{2} \mathbf{M} \mathbf{F}^{-2}+\mathbf{F}^{3} \mathbf{M} \mathbf{F}^{-3}+\mathbf{F}^{4} \mathbf{M} \mathbf{F}^{-4}=\mathbf{K}$

where we have used $\mathbf{F}^{4}=\mathbf{I}$ in the last line.

Next, we use the fact that $\mathbf{F}$ is a simple matrix (since it is unitary); therefore, $\mathbf{F}$ can be diagonalized or block diagonalized in case of repeated eigenvalues. Since $\mathbf{F}$ has only four different eigenvalues, $\{1,-1, j,-j\}, \mathbf{F}$ can be block-diagonalized into four blocks, each block having the size matching the multiplicity of the associated eigenvalue. Two matrices are said to commute if they have the same eigenspaces and the eigendecomposition of the matrices coincides in the same block diagonal-structure. Then the total degrees of freedom in the selection of DFT-commuting matrices is $\rho_{1}^{2}+\rho_{-1}^{2}+\rho_{j}^{2}+\rho_{-j}^{2}$, where $\rho_{k}^{2}$ is the multiplicity of the eigenvalue $k, k \in\{1,-1, j,-j\}$ [8, p. 419].

We now present a theorem showing that the degrees of freedom in the generation of $\mathbf{K}$ matrices according to (3) matches the total degrees of freedom in the generation of DFT-commuting matrices.

Theorem 2: The degrees of freedom in the generation of matrix $\mathbf{K}$ is $\rho_{1}^{2}+\rho_{-1}^{2}+\rho_{j}^{2}+\rho_{-j}^{2}$, where $\rho_{k}$ is the multiplicity of the eigenvalues of the $N \times N$ DFT matrix.
Proof: By stacking the columns of $\mathbf{K}$ into a vector, $\operatorname{vec}(\mathbf{K})$, we get

$$
\begin{aligned}
& \operatorname{vec}(\mathbf{K}) \\
& \quad \underbrace{\left(\mathbf{I} \otimes \mathbf{I}+\left(\mathbf{F}^{-1}\right)^{T} \otimes \mathbf{F}+\left(\mathbf{F}^{-2}\right)^{T} \otimes \mathbf{F}^{\mathbf{2}}+\left(\mathbf{F}^{-3}\right)^{T} \otimes \mathbf{F}^{\mathbf{3}}\right)}_{\mathbf{A}}
\end{aligned}
$$

where $\otimes$ denotes the Kronecker product. The rank of matrix $\mathbf{A}$ determines the degrees of freedom in the construction of the $\mathbf{K}$ matrix. We shall determine the rank of $\mathbf{A}$ through its eigenvalues.

It is known that the matrix $p(\mathbf{Y} ; \mathbf{X})=\sum_{k=0}^{3} \mathbf{Y}^{k} \otimes \mathbf{X}^{k}$ has the eigenvalues $p\left(\lambda_{y} ; \lambda_{x}\right)=\sum_{k=0}^{3} \lambda_{y}^{k} \lambda_{x}^{k}$, where $\lambda_{x}$ and $\lambda_{y}$ are any eigenvalues of $\mathbf{X}$ and $\mathbf{Y}$ matrices, [8, p. 411]. The associated eigenvector for the eigenvalue $p\left(\lambda_{y} ; \lambda_{x}\right)$ is $\mathbf{e}_{\lambda_{y}} \otimes \mathbf{e}_{\lambda_{x}}$, where $\mathbf{e}_{\lambda_{x}}$ and $\mathbf{e}_{\lambda_{y}}$ are eigenvectors of $\mathbf{X}$ and $\mathbf{Y}$ corresponding to the eigenvalues $\lambda_{x}$ and $\lambda_{y}$, respectively.

Then the eigenvalues of $p\left(\mathbf{F}^{*} ; \mathbf{F}\right)$ are $\sum_{k=0}^{3}\left(\lambda_{m}^{*}\right)^{k} \lambda_{n}^{k}$ with the associated eigenvector $\mathbf{e}_{\lambda_{m}} \otimes \mathbf{e}_{\lambda_{n}}$. Since $\lambda_{n}, \lambda_{m}$ is limited to the primitive fourth roots of unity, i.e., $\{1,-1, j,-j\}$, the eigenvalues of $p\left(\mathbf{F}^{*} ; \mathbf{F}\right)$ are zero unless $\lambda_{n} \neq \lambda_{m}$. The only nonzero eigenvalue of $p\left(\mathbf{F}^{*} ; \mathbf{F}\right)$ is four and it occurs when both $\mathbf{e}_{\lambda_{m}}$ and $\mathbf{e}_{\lambda_{n}}$ are associated with the same eigenvalue, i.e., when both vectors belong to the same eigenspace. The number of independent eigenvectors in each invariant sub-space is the eigenvalue multiplicity number, $\rho_{k}$. Therefore, the total number eigenvectors with nonzero eigenvalue, or the rank of $\mathbf{A}$, is $\rho_{1}^{2}+\rho_{-1}^{2}+$ $\rho_{j}^{2}+\rho_{-j}^{2}$.

We now further simplify the structure of commuting matrices. Let $J_{c}$ be the cyclic coordinate inversion operation. That is, with the application of $\mathbf{J}_{\mathbf{c}}$ to vector $\mathbf{x}=[x(0), x(1), x(2), \cdots x(N-$ $1)]^{T}$, we get $[x(0), x(N-1), x(N-2), \cdots x(1)]^{T}$. We know that $\mathbf{F}^{2}=\mathbf{F}^{-2}=\mathbf{J}_{\mathbf{c}}$, replacing $\mathbf{F}^{2}$ with $\mathbf{J}_{\mathbf{c}}$ in (3), we get:

$$
\mathbf{K}=\mathbf{M}+\mathbf{J}_{\mathbf{c}} \mathbf{M} \mathbf{J}_{\mathbf{c}}+\mathbf{F} \mathbf{M F}{ }^{-1}+\mathbf{F} \mathbf{J}_{\mathbf{c}} \mathbf{M} \mathbf{J}_{\mathbf{c}} \mathbf{F}^{-1} .
$$

This leads to the following structure for the commuting matrix K:

$$
\mathbf{K}=\left[\begin{array}{ll}
k_{11} & \mathbf{k}_{12} \\
\mathbf{k}_{21} & \mathbf{K}_{22}
\end{array}\right]
$$

where $k_{11}$ is a scalar, $\mathbf{k}_{12}, \mathbf{k}_{21}$ are row and column vectors of length $N-1$, and $\mathbf{K}_{22}$ is a centro-symmetric matrix of size $(N-1) \times(N-1)$.

In the literature, there are two well-known DFT-commuting matrices. The commuting $\mathbf{S}$ matrix introduced in [6] has a generating $\mathbf{M}$ matrix that is a circular convolution matrix of second differencing operation. The commuting $\mathbf{T}$ matrix introduced in [7] has a non-Toeplitz structure, but it has also been shown to be related to second differentiation [3]. Both matrices are in the described structure, as expected. In [2], eigendecomposition of the commuting matrix $\mathbf{S}$ has been studied in detail; this study can be extended by using eigendecomposition properties of centro-symmetric matrices [9]. 


\section{IV. $\mathbf{S}_{2 k}$ MATRICES}

In the classical text of Hildebrand, [10, p. 125], some approximations to second derivative have been given as follows:

$$
\begin{aligned}
& f^{\prime \prime}\left(x_{k}\right)=\frac{\delta^{2} f_{k}}{h^{2}}+O\left(h^{2}\right) \\
& f^{\prime \prime}\left(x_{k}\right)=\frac{\delta^{2} f_{k}-\frac{1}{12} \delta^{4} f_{k}}{h^{2}}+O\left(h^{4}\right)
\end{aligned}
$$

where $\delta^{2} f_{k}=f_{k+1}-2 f_{k}+f_{k-1}$ represents the second central differencing. In [2], (8) has been employed for the second derivative approximation to generate an $O\left(h^{2}\right)$ approximation to the Hermite-Gaussian generating differential equation. Finer approximations have also been suggested. For an $O\left(h^{4}\right)$ approximation, we can use

$$
\begin{array}{r}
\left(\delta^{2}-\frac{1}{12} \delta^{4}\right) f[n]=-\frac{1}{12} f[n+2]+\frac{4}{3} f[n+1]-\frac{5}{2} f[n] \\
+\frac{4}{3} f[n-1]-\frac{1}{12} f[n-2] .
\end{array}
$$

Expressing convolution of (10) in matrix form, we get a finite dimensional operator approximating the second derivative as follows:

$$
\mathbf{M}=\left[\begin{array}{ccccccc}
-\frac{5}{2} & \frac{4}{3} & -\frac{1}{12} & 0 & 0 & -\frac{1}{12} & \frac{4}{3} \\
\frac{4}{3} & -\frac{5}{2} & \frac{4}{3} & -\frac{1}{12} & 0 & 0 & -\frac{1}{12} \\
-\frac{1}{12} & \frac{4}{3} & -\frac{5}{2} & \frac{4}{3} & -\frac{1}{12} & 0 & 0 \\
0 & -\frac{1}{12} & \frac{4}{3} & -\frac{5}{2} & \frac{4}{3} & -\frac{1}{12} & 0 \\
0 & 0 & -\frac{1}{12} & \frac{4}{3} & -\frac{5}{2} & \frac{4}{3} & -\frac{1}{12} \\
-\frac{1}{12} & 0 & 0 & -\frac{1}{12} & \frac{4}{3} & -\frac{5}{2} & \frac{4}{3} \\
\frac{4}{3} & -\frac{1}{12} & 0 & 0 & -\frac{1}{12} & \frac{4}{3} & -\frac{5}{2}
\end{array}\right] .
$$

Inserting the circular convolution matrix $\mathbf{M}$ in (11) into the defining relation for DFT-commuting matrices, which is in (6), we get a DFT-commuting matrix $\mathbf{K}$. The important fact is that the resulting $\mathbf{K}$ matrix not only commutes with DFT but also is an $O\left(h^{4}\right)$ approximation to the Hermite-Gaussian generating differential equation. This is true since $\mathbf{J}_{\mathbf{c}} \mathbf{M} \mathbf{J}_{\mathbf{c}}=\mathbf{M}$ and $\mathbf{K}$ reduces to $\mathbf{K}=2\left(\mathbf{M}+\mathbf{F} \mathbf{M} \mathbf{F}^{-1}\right)$, an $O\left(h^{4}\right)$ approximation of the Hermite-Gaussian generating differential equation (2).

The method for the fourth-order approximation can now be immediately generalized to higher orders. The formula for $O\left(h^{2 k}\right)$ approximation to second derivative can be derived as follows [11]:

$f^{\prime \prime}\left(x_{k}\right)=\left(\sum_{m=1}^{k}(-1)^{(m-1)} \frac{2[(m-1) !]^{2}}{(2 m) !} \delta^{2 m}\right) \frac{f_{k}}{h^{2}}+O\left(h^{2 k}\right)$.

The $O\left(h^{2 k}\right)$ approximation formulas given above used together with the commuting matrix structure in (6) result in DFT commuting, Hermite-Gaussian approximating matrices that are called $\mathbf{S}_{2 k}$ matrices.

We note that utilization of higher order matrices comes at no additional computational cost, since the commuting matrices $\mathbf{S}$, $\mathbf{T}$, and $\mathbf{S}_{2 k}$ do not have any known fast eigenvector calculation

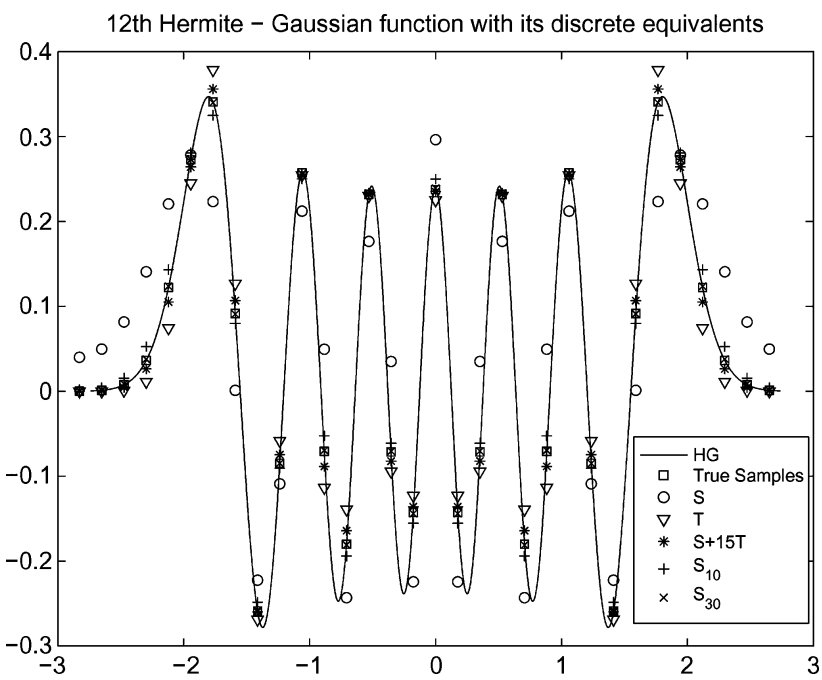

Fig. 1. Comparison of twelfth Hermite-Gaussian with its discrete equivalents using different approximation techniques.

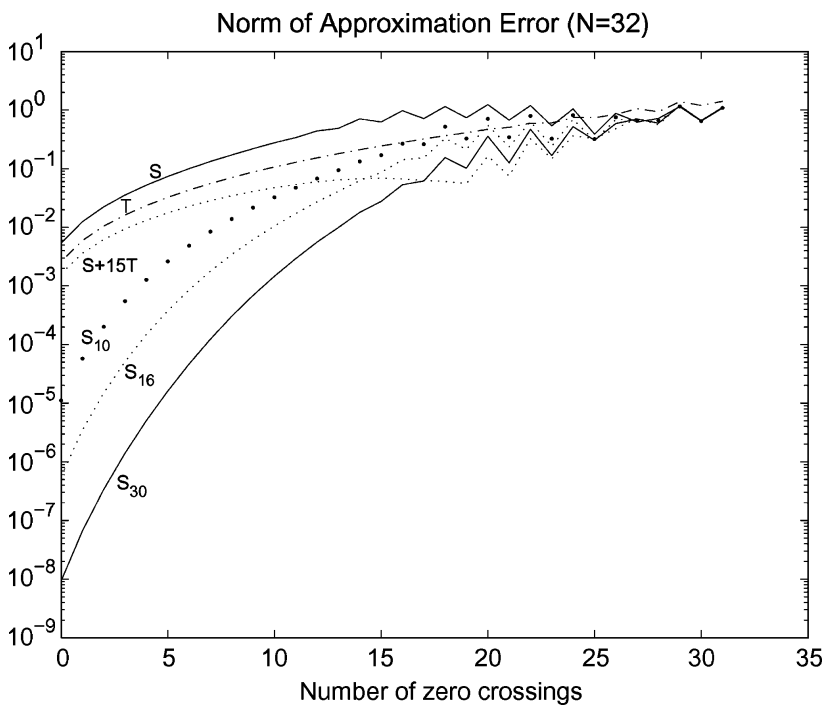

Fig. 2. Comparison of error norms with different approximation techniques.

methods. The general methods for the banded matrices are used for eigenvector calculation.

\section{NUMERICAL COMPARISONS}

In this section, we present some numerical comparison results to show the approximation power of higher order matrices and compare them with the matrices proposed in the literature.

Fig. 1 shows the twelfth Hermite-Gaussian function and its 32-point discrete representations calculated using different matrices. The representations shown are obtained through the $\mathbf{S}$ matrix [2]; the $\mathbf{T}$ matrix [3], [7]; the $\mathbf{S}+15 \mathbf{T}$ matrix [3]; and higher order approximation matrices $\mathbf{S}_{10}$ and $\mathbf{S}_{30}$. As can be observed from Fig. 1, the $\mathbf{S}$ matrix combined with $\mathbf{T}$ produces remarkably good approximations almost at the approximation level of $\mathbf{S}_{10}$. However, clearly $\mathbf{S}_{30}$ produces uniformly better approximations, much closer to the true samples at every sampling point. The approximation error norm is 0.0596 and 0.0056 for $\mathbf{S}+15 \mathbf{T}$ and $\mathbf{S}_{30}$ matrices, respectively.

In Fig. 2, the approximation error between true samples of Hermite-Gaussians and their discrete equivalents are plotted for 
$N=32$. As expected, the higher order matrices produce much finer approximations. The performance of all methods degrade as the order Hermite-Gaussian functions increase. We also note from Fig. 2 that $\mathbf{S}+15 \mathbf{T}$ matrix produces good approximations, at times better than the ones of $\mathbf{S}_{30}$ to Hermite-Gaussian functions for higher order Hermite-Gaussians. Therefore, the $\mathbf{S}+15 \mathbf{T}$ matrix can be used to approximate higher order Hermite-Gaussian functions when these functions are discretized individually. Additional comparisons for $N=\{16,64,128\}$ can be found in [11].

\section{CONCLUSIONS}

In this letter, we have showed that higher order approximation matrices suggested earlier in [2] provide much better approximations than the approximations recently suggested for the improvement of fractional Fourier transform calculation. Here, we present an analysis on the space of DFT-commuting matrices. In the space of commuting matrices, we isolate $\mathbf{S}_{2 k}$ matrices and show that they can be used to accurately approximate Hermite-Gaussian functions and therefore fractional Fourier transforms.

\section{REFERENCES}

[1] H. M. Ozaktas, Z. Zalevsky, and M. A. Kutay, The Fractional Fourier Transform with Applications in Optics and Signal Processing. New York: Wiley, 2000

[2] C. Candan, M. A. Kutay, and H. M. Ozaktas, "The discrete fractional Fourier transform," IEEE Trans. Signal Process., vol. 48, no. 5, pp. 1329-1337, May 2000.

[3] S. C. Pei, W. L. Hsue, and J. J. Ding, "Discrete fractional Fourier transform based on new nearly tridiagonal commuting matrices," IEEE Trans. Signal Process., vol. 54, no. 10, pp. 3815-3828, Oct. 2006.

[4] S. Clary and D. H. Mugler, "Shifted Fourier matrices and their tridiagonal commutors," SIAM J. Anal. Appl., vol. 24, pp. 809-821, 2003.

[5] G. Strang, "The discrete Cosine transform," SIAM Rev., vol. 41, pp. 135-147, 1999.

[6] B. W. Dickinson and K. Steiglitz, "Eigenvectors and functions of the discrete Fourier transform," IEEE Trans. Acoust., Speech, Signal Process., vol. ASSP-30, no. 1, pp. 25-31, Feb. 1982.

[7] F. A. Grunbaum, "The eigenvectors of the discrete Fourier transform: A version of the Hermite functions," J. Math. Anal. Appl., vol. 88, pp. 355-363, 1982.

[8] P. Lancaster and M. Tismenetsky, The Theory of Matrices. New York: Academic, 1985.

[9] J. R. Weaver, "Centrosymmetrix (cross-symmetric) matrices, their basic properties, eigenvalues, and eigenvectors," Amer. Math. Monthly, vol. 92, pp. 711-717, 1985.

[10] F. Hildebrand, Finite-Difference Equations and Simulations. Englewood Cliffs, NJ: Prentice-Hall, 1968.

[11] C. Candan, "Discrete fractional Fourier transform," M.S. thesis, Bilkent Univ., Ankara, Turkey, 1998. 